\title{
On continuously defective elastic crystals
}

\author{
Marek Z. Elzanowski and Serge Preston
}




\title{
ON CONTINUOUSLY DEFECTIVE ELASTIC CRYSTALS
}

\author{
MAREK Z. ELŻANOWSKI AND SERGE PRESTON
}

\begin{abstract}
We analyze mathematical underpinnings of Davini's theory of defective crystals [1] when the defectiveness of a kinematic state may be material point dependent. We show how the underlying space can be identified with a suitably chosen homogeneous space and how the uniformly defective structure is just a special case.
\end{abstract}

2000 Mathematics Subject Classification: 53Z05, 74B99

Keywords: defects, defective elastic crystals, homogeneous spaces

\section{INTRODUCTION}

The kinematic theory of continuously defective elastic crystal bodies was originally proposed by Davini [1] and developed over the last two decades by him and Parry, and his collaborators (see, for example, $[2,3,7]$ ). The key assumption of this approach is that the state of a defective (continuous) crystal is defined by three linearly independent and smoothly varying over the body, $\mathbb{R}^{3}$ in our case, lattice vector fields which are to represent an averaged micro (atomic) structure. The defectiveness of such a state is described by the dislocation density tensor, an object which measures the first order interrelations of the given lattice vector fields.

The main focus of the theory has been so far on uniform defective crystals, that is, the states characterized by the property that the dislocation density tensor is constant throughout the body. The reason for this is that when the given vector fields are such that the corresponding dislocation density tensor is position independent, the underlying space (the body) can be equipped with a Lie group structure in such a way that the lattice vector fields are right invariant under its action on itself, [7]. The availability of this "additional" structure allows one to use the power of the theory of Lie groups and algebras to analyze in a systematic way the properties of such uniform defective crystal states, in particular, the question of symmetries, both local and global, discrete and continuous (see for example [8]). In contrast, when the lattice vector fields are such that the dislocation density tensor is material point dependent, the Lie group structure is no longer available.

In this short note, we look closer at the states with a non-uniform dislocation density tensor. We show that although the underlying space $\mathbb{R}^{3}$ cannot indeed be assigned a Lie group structure, it can be identified with the properly defined homogeneous 
space the total space of which is a Lie group. The Lie algebra of this group is homomorphic to a Lie subalgebra of smooth vector fields on $\mathbb{R}^{3}$ generated uniquely by the three lattice vector fields. The homogeneous space structure of $\mathbb{R}^{3}$ collapses to a Lie group when the Lie algebra generated by the lattice vector fields is of dimension three and the corresponding dislocation density tensor is base point independent, thus proving that the uniformly defective state is just a special case of a general kinematic state of a defective crystal.

The paper is divided into four short sections. The first two sections are dedicated to the presentation of the foundations of Davini's theory [1] and the analysis of the uniformly defective states, [7]. In Section 3, we deal with the non-uniform case. In the last section, we show how the dislocation density tensor relates to the intrinsic characteristic of the homogeneous space associated with the lattice vector fields.

\section{Continuous elastic CRystals}

Let the kinematic state of a continuous solid crystal body be given by three linearly independent vector fields $l_{i}: \mathbb{R}^{3} \rightarrow T \mathbb{R}^{3}, i=1,2,3$, where $T \mathbb{R}^{3}$ denotes the tangent space to $\mathbb{R}^{3}$. In other words, the state of a continuous elastic crystal is defined be a global smooth section $\mathbf{I}: \mathbb{R}^{3} \rightarrow L\left(\mathbb{R}^{3}\right)$ of the bundle of the linear frames of $\mathbb{R}^{3}$, [5], called a continuous lattice or simply a lattice. Invoking the Euclidean structure of $\mathbb{R}^{3}$, the lattice $\mathbf{l}(x)$ induces the dual frame $\mathbf{d}: \mathbb{R}^{3} \rightarrow L\left(\mathbb{R}^{3}\right)$ such that $d_{i}(x) \cdot l_{j}(x)=\delta_{i j}$, $i, j=1,2,3, x \in \mathbb{R}^{3}$, where $\delta_{i j}$ denotes the usual Kroneker's delta. In this context, the "defectiveness" of the lattice $\mathbf{I}(x)$ can be characterized by the dislocation density tensor (ddt) the components of which are defined by the equations

$$
n(x) S_{i j}(x)=\nabla \wedge d_{i}(x) \cdot d_{j}(x), \quad i, j=1,2,3, \quad x \in \mathbb{R}^{3},
$$

where $n(x):=d_{1}(x) \cdot d_{2}(x) \wedge d_{3}(x)$ is the lattice volume element. Looking closer at the properties of the dislocation density tensor, we note that if the defining frame field $\mathbf{l}(x)$ is holonomic (integrable), the corresponding dislocation density tensor vanishes everywhere. In fact, the opposite is also true. That is, if $S_{i j}(x)$ is identically zero, the lattice $\mathbf{I}(x)$ it represents is holonomic, [2]. In particular, the dislocation density tensor of the ideal lattice defined by a constant orthonormal frame $\mathbf{e}(x)$ vanishes identically.

Having two crystalline structures, say $\mathbf{I}(x)$ and $\widetilde{\mathbf{I}}(x)$, we state that they are elastically related if there exists a diffeomorphism $\phi: \mathbb{R}^{3} \rightarrow \mathbb{R}^{3}$ such that

$$
\tilde{l}_{i}(\phi(x))=\phi_{*}\left(l_{i}(x)\right), \quad i=1,2,3, \quad x \in \mathbb{R}^{3}
$$

where $\phi_{*}: T \mathbb{R}^{3} \rightarrow T \mathbb{R}^{3}$ denotes the tangent map of $\phi$. Thus, any diffeomorphism of $\mathbb{R}^{3}$, when applied to a continuous lattice via (1.2), induces an elastically related structure. It is not true, however, that any two (smooth) crystalline structures are elastically related unless additional conditions are met. Indeed, as shown in [1], one of the properties of an elastic deformation is that it preserves the defectiveness of the given continuous lattice. Namely, given a diffeomorphism $\phi: \mathbb{R}^{3} \rightarrow \mathbb{R}^{3}$, the lattice 
$\mathbf{I}(x)$, and the elastically induced lattice $\widetilde{\mathbf{I}}(\phi(x))=\phi_{*}(\mathbf{l}(x))$,

$$
\tilde{S}_{i j}(\phi(x))=S_{i j}(x), \quad i, j=1,2,3, \quad x \in \mathbb{R}^{3}
$$

where $\tilde{S}_{i j}(x)$ are the components of the dislocation density tensor of the new structure*.

The ideal lattice, in fact any holonomic frame field, is an example of a continuous elastic crystal with a uniform (point independent) dislocation density tensor. More generally, a continuous lattice is called uniformly defective if its dislocation density tensor $S_{i j}(x)$ is material point independent. It is easy to see from the equation (1.3) that if two uniformly defective lattices are elastically related, they have the same dislocation density tensor. The reader should, however, be cautioned that two uniformly defective lattices with the same dislocation density tensor are not guaranteed to be elastically related. Indeed, such lattices may be related by the neutral deformation [1], which preserves defectiveness of a lattice but is not elastic in the sense that it does not come from a diffeomorphism of the underlying space $\mathbb{R}^{3}$ in our case.

In the next section, we shall look closer at the uniformly defective continuous lattices summarizing how the fact that the dislocation density tensor if uniform allows one to introduce a nontrivial group structure on the underlying space $\mathbb{R}^{3}$.

\section{UNIFORMLY DEFECTIVE STRUCTURES}

Consider a uniformly defective continuous lattice $\mathbf{I}(x)$ with the dislocation density tensor $S_{i j}$ and such that $l_{i}(\mathbf{0})=\mathbf{e}_{i}, i=1,2,3$, at the origin $\mathbf{0} \in \mathbb{R}^{3}$. Motivated by the fact that $S_{i j}$ is constant and that any elastic deformation preserves it, we ask if given an arbitrary point $u \in \mathbb{R}^{3}$ there exists a diffeomorphism $\psi(\cdot, u): \mathbb{R}^{3} \rightarrow \mathbb{R}^{3}$ such that $\psi(\mathbf{0}, u)=u$ and

$$
l_{i}(\psi(x, u))=\nabla_{1} \psi(x, u) l_{i}(x), \quad i=1,2,3, \quad x, u \in \mathbb{R}^{3}
$$

where $\nabla_{1} \psi(\cdot, u)$ denotes the Jacobian of $\psi$ with respect to the (first) variable. The assumption that the dislocation density tensor $S_{i j}$ is constant turns out to be an integrability condition for the system of equations (2.1), [7], guaranteeing that there is a smooth and invertible (in each variable separately) solution $\psi: \mathbb{R}^{3} \times \mathbb{R}^{3} \rightarrow \mathbb{R}^{3}$ which is associative and such that for every $x \in \mathbb{R}^{3}$ there exists $x^{-1} \in \mathbb{R}^{3}$ with the property that

$$
\psi\left(x, x^{-1}\right)=\psi\left(x^{-1}, x\right)=\mathbf{0},
$$

where

$$
\psi(\mathbf{0}, x)=\psi(x, \mathbf{0})=x .
$$

*A homogeneous deformation $\phi(x)=\mathbf{m} x$ is a simple example of an elastic deformation, where $\mathbf{\text { a }} \equiv\left\{\gamma_{i}^{j}\right\}$ is a non-singular $3 \times 3$ matrix. Indeed, as easily confirmed by the direct calculations, the deformed lattice $\hat{l}_{i}(x)=\gamma_{i}^{j} l_{j}\left(\mathbf{\square}^{-1} x\right)$ has the dislocation density tensor $\widehat{S}_{i j}(x)=S_{i j}\left(\mathbf{\square}^{-1} x\right)$ for every $x \in \mathbb{R}^{3}$. 
In other words, any uniformly defective lattice is elastically self-similar. Conversely, if, for some continuous lattice $\mathbf{l}(x)$, the system of equations (2.1) has an associative solution $\psi$ satisfying (2.2) and (2.3), the lattice must be uniformly defective.

The associative solution $\psi$ to the system of equations (2.1) can be viewed as a group multiplication on the underlying space $\mathbb{R}^{3}$ with the identity element at the origin $\mathbf{0} \in \mathbb{R}^{3}$. In fact, as the mapping $\psi$ is smooth and invertible in each argument, it introduces a Lie group structure on $\mathbb{R}^{3}$ such that the vector fields $l_{i}(x), i=1,2,3$ are right invariant under its action. This implies that the algebra of vectors fields generated by $l_{i}(x), i=1,2,3$, with the standard Lie bracket $\left[l_{i}, l_{j}\right]:=\left(l_{i} \cdot \nabla\right) l_{j}-$ $\left(l_{j} \cdot \nabla\right) l_{i}$ is isomorphic to the Lie algebra of the left invariant vector fields on $\mathbb{R}^{3}$ viewed as a group with the multiplication $\psi: \mathbb{R}^{3} \times \mathbb{R}^{3} \rightarrow \mathbb{R}^{3}$. Let $C_{k i j}$ denote the corresponding Lie algebra constants, that is, $\left[l_{i}, l_{j}\right]=C_{k i j} l_{k}, i, j, k=1,2,3$, where the summation convention over the repeated indices is enforced. It can be shown, [3], that the dislocation density tensor is such that

$$
\epsilon_{j k l} S_{i j}=C_{i k l}
$$

where $\epsilon_{j k l}$ is the classical alternating tensor.

To end this section, we present a simple example illustrating the concepts introduced so far.

Example 1. Let us consider a continuous lattice defined by the frame

$$
l_{1}(x)=\mathbf{e}_{1}, \quad l_{2}(x)=\mathbf{e}_{2}, \quad l_{3}(x)=x_{1} \mathbf{e}_{1}+x_{2} \mathbf{e}_{2}+\mathbf{e}_{3} .
$$

As the Lie brackets are $\left[l_{1}, l_{2}\right]=0,\left[l_{1}, l_{3}\right]=l_{1}$ and $\left[l_{2}, l_{3}\right]=l_{2}$, the only non-zero Lie algebra constants are $C_{113}=C_{223}=1$. Hence, the dislocation density tensor

$$
S_{i j}=\left(\begin{array}{ccc}
0 & 1 & 0 \\
-1 & 0 & 0 \\
0 & 0 & 0
\end{array}\right) \text {. }
$$

To find a suitable group operation $\psi: \mathbb{R}^{3} \times \mathbb{R}^{3} \rightarrow \mathbb{R}^{3}$, we must solve the system of equations (2.1). It can easily be shown that

$$
\psi(u, v)=u+\left(0,0, v_{3}\right)+e^{u_{3}}\left(v_{1}, v_{2}, 0\right), \quad u, v \in \mathbb{R}^{3}
$$

is indeed a solution. Calculating its Jacobian with respect to its first variable, we obtain

$$
\nabla_{1} \psi(u, v)=\left(\begin{array}{ccc}
1 & 0 & v_{1} e^{u_{3}} \\
0 & 1 & v_{2} e^{u_{3}} \\
0 & 0 & 1
\end{array}\right)
$$

confirming, that the vector fields generating the given lattice are right invariant under the action of this group. 


\section{NON-UNIFORMLY DEFECTIVE STRUCTURES}

We start our analysis of crystal structures which may not be uniformly defective by looking at a specific example of a continuum lattice with a variable dislocation density tensor.

Example 2. Consider the crystalline structure of an elastic body given by three linearly independent vector fields

$$
l_{1}(x)=\mathbf{e}_{1}, \quad l_{2}(x)=\mathbf{e}_{2}-x_{2} \mathbf{e}_{1}, \quad l_{3}(x)=x_{1} \mathbf{e}_{1}+x_{2} \mathbf{e}_{2}+\mathbf{e}_{3}, \quad x \in \mathbb{R}^{3} .
$$

Using the definition of the dislocation density tensor (1.1), we see that it is position dependent as

$$
S_{i j}(x)=\left(\begin{array}{ccc}
-x_{2} & 1 & 0 \\
-1 & 0 & 0 \\
0 & 0 & 0
\end{array}\right), \quad x=\left(x_{1}, x_{2}, x_{3}\right) \in \mathbb{R}^{3} .
$$

Looking back at the identity (2.4), we conclude that either the relation between the Lie algebra constants and the dislocation density tensor is no longer valid and/or the given vector fields do not generate a 3-dim Lie algebra. Indeed, calculating the corresponding Lie brackets, one obtains that

$$
\left[l_{1}(x), l\left({ }_{2}(x)\right]=0, \quad\left[l_{1}(x), l_{3}(x)\right]=l_{1}(x), \quad\left[l_{2}(x), l_{3}(x)\right]=\mathbf{e}_{2}\right.
$$

where the vector field $\mathbf{e}_{2} \equiv l_{2}(x)+x_{2} l_{1}(x)$ cannot be represented as a linear combination of the given vector fields (3.1). Investigating this further, one discovers that as

$$
\left[\mathbf{e}_{2}, l_{1}(x)\right]=0, \quad\left[\mathbf{e}_{2}, l_{2}(x)\right]=-l_{1}(x), \quad\left[\mathbf{e}_{2}, l_{3}(x)\right]=\mathbf{e}_{2},
$$

the smallest Lie algebra of vector fields on $\mathbb{R}^{3}$ the given frame field belongs to is the four dimensional Lie algebra generated by

$$
\mathbf{e}_{1}, \mathbf{e}_{2}, \mathbf{e}_{2}-x_{2} \mathbf{e}_{1}, x_{1} \mathbf{e}_{1}+x_{2} \mathbf{e}_{2}+\mathbf{e}_{3} \text {. }
$$

Thus, it appears that no group structure can be given to the underlying space $\mathbb{R}^{3}$. However, one may ask if there is an algebraic or a differential structure which $\mathbb{R}^{3}$ can be equipped with, above and beyond its natural Euclidean structure. Moreover, one wonders how the dislocation density tensor relates to any intrinsic characteristics of such a structure ${ }^{\dagger}$. We shall investigate these questions in the sequel.

Consider a continuous lattice defined by a frame field $l: \mathbb{R}^{3} \rightarrow L\left(\mathbb{R}^{3}\right)$ such that $l_{i}(\mathbf{0})=\mathbf{e}_{i}, i=1,2,3$. We assume that the lattice fields $l_{i}(x), i=1,2,3$, generate a $k$-dimensional Lie subalgebra, say $\mathfrak{l}$, of the algebra $\mathcal{X}\left(\mathbb{R}^{3}\right)$ of all smooth vector fields on $\mathbb{R}^{3}$, where $3 \leq k<\infty$. Hence, the lattice algebra $\mathfrak{l}$ is spanned by the vector fields, say $\mathfrak{l}_{1}, \mathfrak{l}_{2}, \ldots, \mathfrak{l}_{k}$, where for the consistency of our presentation, the vector fields are ordered so that $\mathfrak{l}_{i}=l_{i}(x), i=1,2,3$, unless stated otherwise. We

\footnotetext{
${ }^{\dagger}$ Note that the relation (2.4) is still valid if the Lie algebra constants are replaced by the variable coefficients $\widehat{C}_{k i j}(x)$ such that $\left[l_{i}, l_{j}\right]=\widehat{C}_{k i j}(x) l_{k}$. See also Proposition 3.
} 
postulate also that all generators of the subalgebra $\mathfrak{l}$ are complete vector fields on the manifold $\mathbb{R}^{3}$, which implies that the algebra $\mathfrak{l}$ consists entirely of complete vector fields, [4]. Moreover, there exists a Lie group acting on $\mathbb{R}^{3}$ the Lie algebra of which is isomorphic to $\mathfrak{l},[4,6]$.

Theorem 1. Consider a continuous lattice defined by three linearly independent smooth vector fields $l_{i}: \mathbb{R}^{3} \rightarrow T \mathbb{R}^{3}, i=1,2,3$. Let $\mathfrak{l} \subset \mathcal{X}\left(\mathbb{R}^{3}\right)$ be the smallest algebra of vector fields containing the given lattice vector fields. Assume that $\mathfrak{l}$ is finite-dimensional and complete. Then, there exists a simply connected Lie group $G$ contained in $\operatorname{Diff}\left(\mathbb{R}^{3}\right)$ as an abstract subgroup and such that the natural action $\Lambda: G \times \mathbb{R}^{3} \rightarrow \mathbb{R}^{3}$ of the group $G$ on $\mathbb{R}^{3}$ is smooth and the algebra $\mathfrak{l}$ is homomorphic to the Lie algebra, say $\mathfrak{g}$, of the group $G$.

Given the smooth action $\Lambda$, there exists a homomorphism $\lambda: G \rightarrow \operatorname{Diff}\left(\mathbb{R}^{3}\right)$ from the group $G$ into the group of all diffeomorphisms of $\mathbb{R}^{3}$ such that

$$
\lambda(a)(x)=\Lambda(a, x), \quad a \in G, x \in \mathbb{R}^{3} .
$$

If, in addition, the action $\Lambda$ is effective, the homomorphism $\lambda$ identifies the group $G$ with a subgroup, say $\lambda(G) \subset \operatorname{Diff}\left(\mathbb{R}^{3}\right)$. Given $x \in \mathbb{R}^{3}$, consider a smooth mapping $\Lambda(x): G \rightarrow \mathbb{R}^{3}$ such that

$$
\Lambda(x)(g):=\Lambda(g, x)
$$

for any $g \in G$. The mapping $\Lambda(x)$ maps the group $G$ onto the orbit $G(x)$ of the point $x$ (under the action $\Lambda$ ). Indeed, $y \in G(x)$ if and only if there exists $a \in G$ such that $y=\Lambda(x)(a) \equiv \Lambda(a, x)$. Moreover, the mapping $\Lambda(x)$ is a morphism (but not necessarily an isomorphism) of the action of $G$ on itself (by left translations) into the action of $\Lambda$ on $\mathbb{R}^{3}$. Correspondingly, there exists a relation between the Lie algebra $\mathfrak{g}$ and the algebra of all smooth vector fields $\mathcal{X}\left(\mathbb{R}^{3}\right)$. To illustrate this fact, let us define the map $d \lambda: \mathfrak{g} \rightarrow \mathcal{X}\left(\mathbb{R}^{3}\right)$ by requiring that

$$
d \lambda(\mathfrak{v})(x):=d_{e} \Lambda(x)(\mathfrak{v})
$$

for any $\mathfrak{v} \in \mathfrak{g}$ and any $x \in \mathbb{R}^{3}$, where $d_{e} \Lambda(x)$ denotes the tangent map of $\Lambda(x)$ at the identity $e \in G$. Note that, by (3.8), $d \lambda(\mathfrak{v})$ is a smooth vector field on $\mathbb{R}^{3}$ while $d_{e} \Lambda(x)(\mathfrak{g})$ is a subset of the tangent space $T_{x} \mathbb{R}^{3}$. In addition, [4],

Proposition 1. The mapping $d \lambda: \mathfrak{g} \rightarrow \mathcal{X}\left(\mathbb{R}^{3}\right)$ is a homomorphism of Lie algebras. In fact, $d \lambda(\mathfrak{g})=\mathfrak{l}$.

Remark 1 . The action $\Lambda$ is a generalization of the action $\psi$ of $\mathbb{R}^{3}$ on itself when the lattice is uniformly defective. Indeed, to illustrate this claim, let us revisit Example 1. There, we shall look at $\mathbb{R}^{3}$ both as a Lie group with the Lie algebra $\mathfrak{g} \equiv \mathbb{R}^{3}$ generated by the standard basis $\left\{\mathbf{e}_{1}, \mathbf{e}_{2}, \mathbf{e}_{3}\right\}$, and as the space (manifold) the group is acting on. The action $\Lambda$ of the additive group $\mathbb{R}^{3}$ on the space $\mathbb{R}^{3}$ is given by

$$
\Lambda(a, u)=\left(a_{1}+u_{1} e^{a_{3}}, a_{2}+u_{2} e^{a_{3}}, a_{3}+u_{3}\right)
$$


where for the clarity of the presentation, $a \in \mathbb{R}^{3}$ denotes a group element while $u \in \mathbb{R}^{3}$ is a point in the space acted upon. To identify the homomorphism $d \lambda$ defined by (3.8), we calculate that

$$
d_{0} \Lambda(u)=\left.d_{a} \Lambda(u)\right|_{a=0}=\left(\begin{array}{ccc}
1 & 0 & u_{1} e^{a_{3}} \\
0 & 1 & u_{2} e^{a_{3}} \\
0 & 0 & 1
\end{array}\right)_{\left.\right|_{a=0}}=\left(\begin{array}{ccc}
1 & 0 & u_{1} \\
0 & 1 & u_{2} \\
0 & 0 & 1
\end{array}\right) .
$$

Hence, given an arbitrary element $\mathfrak{v}=\left(\mathfrak{v}_{1}, \mathfrak{v}_{2}, \mathfrak{v}_{3}\right)$ of the Lie algebra $\mathfrak{g}$, we have

$$
d \lambda(\mathfrak{v})(u)=d_{0} \Lambda(u)(\mathfrak{v})=\left(\begin{array}{ccc}
1 & 0 & u_{1} \\
0 & 1 & u_{2} \\
0 & 0 & 1
\end{array}\right)\left(\begin{array}{l}
\mathfrak{v}_{1} \\
\mathfrak{v} 2 \\
\mathfrak{v}_{3}
\end{array}\right)=\mathfrak{v}_{1} \mathbf{e}_{1}+\mathfrak{v}_{2} \mathbf{e}_{2}+\mathfrak{v}_{3}\left(\begin{array}{c}
u_{1} \\
u_{2} \\
1
\end{array}\right)
$$

which is exactly the subalgebra $\mathfrak{l} \subset \mathcal{X}\left(\mathbb{R}^{3}\right)$ of example 1 , that is, the corresponding lattice algebra; see (2.5).

Given a $k$-parameter Lie group $G$ acting on $\mathbb{R}^{3}$, where the Lie algebra $\mathfrak{g}$ of $G$ is homomorphic to the lattice algebra $\mathfrak{l}$, consider a point, say $x_{0} \in \mathbb{R}^{3}$, and let $G_{x_{0}}$ be the isotropy group of the action $\Lambda$ at $x_{0}$. That is, let

$$
G_{x_{0}}:=\left\{g \in G: \Lambda\left(g, x_{0}\right)=x_{0}\right\},
$$

where the projection $\Lambda\left(x_{0}\right): G \rightarrow \mathbb{R}^{3}$ given by (3.7) is such that $\Lambda\left(x_{0}\right)\left(G_{x_{0}}\right)=x_{0}$. If the action $\Lambda$ is transitive, the rank of the projection $\Lambda\left(x_{0}\right)$ is constant, [4], which, in turn, allows one to identify $\mathbb{R}^{3}$ with the quotient space $G / G_{x_{0}}$. Indeed, consider the mapping $\widehat{\Lambda\left(x_{0}\right)}: G / G_{x_{0}} \rightarrow \mathbb{R}^{3}$ such that

$$
\widehat{\Lambda\left(x_{0}\right)}\left(h G_{x_{0}}\right)=\Lambda\left(x_{0}\right)(h)=\Lambda\left(h, x_{0}\right) .
$$

where $h G_{x_{0}}$ denotes the left co-set of $G_{x_{0}}$ under the left translation of the group $G$. It can easily be shown that $\widehat{\Lambda\left(x_{0}\right)}$ is a diffeomorphism and that the isotropy group at a point, say $y \in \mathbb{R}^{3}$ such that $y=\Lambda\left(h, x_{0}\right)$ for some $h \in G$, is the conjugate of $G_{x_{0}}$, i. e., if $g_{0} \in G_{x_{0}}$, then

$$
\Lambda\left(h g_{0} h^{-1}, y\right)=\Lambda\left(h g_{0} h^{-1}, \Lambda\left(h, x_{0}\right)\right)=\Lambda\left(h g_{0}, x_{0}\right)=\Lambda\left(h, x_{0}\right)=y .
$$

Summarizing what we have just discussed, we state

Theorem 2. Consider a continuous lattice defined by three linearly independent smooth vector fields $l_{i}: \mathbb{R}^{3} \rightarrow T \mathbb{R}^{3}, i=1,2,3$, where $\mathfrak{l} \subset \mathcal{X}\left(\mathbb{R}^{3}\right)$ is the corresponding lattice algebra. Then, if the induced action $\Lambda: G \times \mathbb{R}^{3} \rightarrow \mathbb{R}^{3}$ is transitive, the underlying space $\mathbb{R}^{3}$ can be identified with the homogeneous space $G / G_{0}$ where the subgroup $G_{0} \subset G$ is the isotropy group of the action $\Lambda$ at the origin of $\mathbb{R}^{3}$.

Note that if the isotropy group $G_{0}$ is trivial and/or a normal subgroup of the group $G$, the homogeneous space $G / G_{0}$ is a group proving that the corresponding continuous lattice is uniformly defective. Note also that, as the isotropy groups at two different points of the underlying space are conjugate of each other, the identification 
(diffeomorphism) $\widehat{\Lambda\left(x_{0}\right)}: G / G_{x_{0}} \rightarrow \mathbb{R}^{3}$ is a base point dependent. Indeed, consider $y \in \mathbb{R}^{3}$ and let $h \in G$ be such that $y=\Lambda\left(h, x_{0}\right)$. Then $\Lambda(y)(g)=\Lambda(g, y)=$ $\Lambda\left(g, \Lambda\left(h, x_{0}\right)\right)=\Lambda\left(g h, x_{0}\right)=\Lambda\left(x_{0}\right)(g h)$ for any $g \in G$. In other words,

$$
\Lambda(y)=\Lambda\left(\Lambda\left(h, x_{0}\right)\right)=\Lambda\left(x_{0}\right) \circ R_{h}: G \rightarrow \mathbb{R}^{3}
$$

where $R_{h}: G \rightarrow G$ denotes here the right translation by $h \in G$.

Example 3. We shall revisit now Example 2 where the continuous lattice $\mathbf{l}$ was given by the frame (3.1) with the corresponding four-dimensional lattice algebra $\mathfrak{l}$ of vector fields on $\mathbb{R}^{3}$ generated by (3.5). Thus, using the fact that the group of affine transformations of $\mathbb{R}^{3}$ can be realized as a subgroup of the general linear group $G L(4, \mathbb{R})$, it is easy to show that the general element $\mathfrak{v}$ of the algebra $\mathfrak{g}$ of the subgroup $G \subset \operatorname{Diff}\left(\mathbb{R}^{3}\right)$ acting on $\mathbb{R}^{3}$ can be represented as

$$
\mathfrak{v}=\left(\begin{array}{cccc}
q & -r & 0 & t \\
0 & q & 0 & s+r \\
0 & 0 & 0 & q \\
0 & 0 & 0 & 0
\end{array}\right), \quad q, r, s, t \in \mathbb{R}
$$

where

$$
\mathfrak{v}=t \mathbf{e}_{1}+s \mathbf{e}_{2}+r\left(\mathbf{e}_{2}-x_{2} \mathbf{e}_{1}\right)+q\left(x_{1} \mathbf{e}_{1}+x_{2} \mathbf{e}_{2}+\mathbf{e}_{3}\right), \quad x_{1}, x_{2} \in \mathbb{R} .
$$

One can also show that the corresponding, but re-parameterized, four parameter subgroup $G$ of $\operatorname{Diff}\left(\mathbb{R}^{3}\right)$ (or rather its $G L(4, \mathbb{R})$ representation) is given by the elements

$$
g(q, r, \alpha, \beta)=\left(\begin{array}{cccc}
e^{q} & -r e^{q} & 0 & \alpha \\
0 & e^{q} & 0 & \beta \\
0 & 0 & 1 & q \\
0 & 0 & 0 & 1
\end{array}\right) .
$$

This means that the smooth action $\Lambda$ of the group $G$ on $\mathbb{R}^{3}$ takes the form

$$
\Lambda\left(g(q, r, \alpha, \beta),\left(u_{1}, u_{2}, u_{3}\right)\right)=\left(\begin{array}{c}
u_{1} e^{q}-u_{2} r e^{q}+\alpha \\
u_{2}+\beta \\
u_{3}+q
\end{array}\right)
$$

where $\left(u_{1}, u_{2}, u_{3}\right) \in \mathbb{R}^{3}$. The corresponding homomorphism $d \lambda$ of the algebras $\mathfrak{g}$ and $\mathfrak{l}$ can now be easily evaluated as

$$
d \lambda(\cdot)(u)=d_{e} \Lambda(u)=\left(\begin{array}{cccc}
u_{1} & -u_{2} & 1 & 0 \\
u_{2} & 1 & 0 & 1 \\
1 & 0 & 0 & 0
\end{array}\right)
$$

where the identity element $e$ of the group $G$ is attained when all the parameters vanish. Note that the isotropy group of the action $\Lambda$ at the origin $\mathbf{0} \in \mathbb{R}^{3}$ is represented 
by

$$
\left(\begin{array}{cccc}
1 & -r & 0 & 0 \\
0 & 1 & 0 & 0 \\
0 & 0 & 1 & 0 \\
0 & 0 & 0 & 1
\end{array}\right)
$$

\section{DISLOCATION DENSITY TENSOR}

When a continuous lattice is uniformly defective, its dislocation tensity tensor $S_{i j}$ can be represented as a linear combination of the Lie algebra constants of the defining lattice frame $l_{i}(x), i=1,2,3$, see (2.4). On the other hand, when the lattice is nonuniform, that is, when the dislocation density tensor is material point dependent, the relation (2.4) is no longer valid. In this section, we shall show how to re-interpret this relation so as to make it a natural generalization of the uniformly defective case.

To this end, note first that the projection $\Lambda(0): G \rightarrow \mathbb{R}^{3}$, which we shall denote by $\Lambda_{0}$, commutes with the left action of $G$ as

$$
\Lambda_{0}(g h)=\Lambda(g h, 0)=\Lambda(g, \Lambda(h, 0))=\Lambda\left(g, \Lambda_{0}(h)\right)
$$

for any $g, h \in G$. Given the lattice subalgebra $\mathfrak{l} \subset \mathcal{X}\left(\mathbb{R}^{3}\right)$, we can lift its generators $l_{i}(x), i=1, \cdots, k$ to the Lie algebra $\mathfrak{g}$ of the group $G$ using the homomorphism $d \lambda$, (3.8). That is, let $\mathfrak{l}_{i} \in \mathfrak{g}$ be such that $d \lambda\left(\mathfrak{l}_{i}\right)=l_{i}(x), i=1, \cdots, k$. Hence, using the relation (3.15), we obtain that

$$
d \lambda\left(\mathfrak{l}_{i}\right)(x)=d_{e} \Lambda(x)\left(\mathfrak{l}_{i}\right)=d_{e}\left(\Lambda_{0} \circ R_{h}\right)\left(\mathfrak{l}_{i}\right)=d_{h} \Lambda_{0} \circ d_{e} R_{h}\left(\mathfrak{l}_{i}\right)
$$

where $x=\Lambda(h, 0)$ and $h \in G$. This proves, in fact, that given the generators $\mathfrak{l}_{i}$ of the Lie algebra $\mathfrak{g}$ and extending them by the right translation to the whole group $G$, one obtains a right-invariant frame field $\widetilde{\mathfrak{l}}_{i}(h):=d_{e} R_{h}\left(\mathfrak{l}_{i}\right)$ such that it projects onto the generators of the lattice algebra l. Namely,

$$
d \Lambda_{0}\left(\tilde{\mathfrak{l}}_{i}(h)\right)=l_{i}(\Lambda(h, 0))
$$

for any $i=1, \cdots, k$ and $h \in G$. In particular, the first three vector fields $\tilde{\mathfrak{l}}_{i}: G \rightarrow$ $T G$, corresponding to the lattice frame $l_{i}: \mathbb{R}^{3} \rightarrow T \mathbb{R}^{3}$, form a 3-dimensional, right invariant, distribution $\tilde{\mathfrak{D}}: G \rightarrow T G$ (of vector spaces) in $T G$. That is,

$$
\tilde{\mathfrak{D}}(h)=d_{e} R_{h}(\tilde{\mathfrak{D}}(e)) .
$$

The projection of the distribution $\tilde{\mathfrak{D}}$ by the tangent map $d \Lambda_{0}$ is, by the definition of $\Lambda_{0}$ and the explicit construction of the frame field $\widetilde{\mathfrak{l}}_{i}(h), i=1,2,3$, the tangent space $T \mathbb{R}^{3}$. This immediately implies

Proposition 2. Let $\Lambda: G \times \mathbb{R}^{3}$ be a transitive action of a Lie group $G$ on $\mathbb{R}^{3}$. Given a complete lattice frame $\mathbf{l}: \mathbb{R}^{3} \rightarrow L\left(\mathbb{R}^{3}\right)$ such that the Lie algebra $\mathfrak{g} \equiv$ $T_{e} G$ is isomorphic to the lattice algebra $\mathfrak{l} \subset \mathcal{X}\left(\mathbb{R}^{3}\right)$, the tangent space $T G$ can be 
decomposed into the direct sum of the right-invariant distribution $\tilde{\mathfrak{D}}$ and the tangent space to the fibers of the projection $G \rightarrow G / G_{0}$. That is, at any point $p \in G$

$$
T_{p} G=\tilde{\mathfrak{D}}(p) \oplus T_{p}\left(p G_{0}\right) .
$$

Moreover, this direct sum is right invariant under the natural action of the isotropy group $G_{0}$.

Indeed, consider an arbitrary $x \in \mathbb{R}^{3}$ and $h \in G$ such that $\Lambda(h, 0)=x$. Knowing that the mapping $\hat{\Lambda}_{0}$, see (3.13), identifying the underlying space $\mathbb{R}^{3}$ with the homogeneous space $G / G_{0}$, is a diffeomorphism, one can easily see that the inverse image $\hat{\Lambda}_{0}^{-1}(x)=h G_{0}$ is a submanifold. Thus, the kernel

$$
\operatorname{ker} d_{h G_{0}} \hat{\Lambda}_{0}=\operatorname{ker} d_{h} \Lambda_{0}=T_{p}\left(p G_{0}\right) .
$$

This and the fact that $d_{h} \Lambda_{0}(\mathfrak{D}(h))=T_{\Lambda(h, 0)} \mathbb{R}^{3}$ prove finally the decomposition (4.5).

In general, the vector space $\mathfrak{D}:=\tilde{\mathfrak{D}}(e) \subset \mathfrak{g}$ is not a subalgebra ${ }^{\S}$, thus, the commutators $\left[\mathfrak{l}_{i}, \mathfrak{l}_{j}\right], i, j=1,2,3$, although in the algebra $\mathfrak{g}$, are not necessarily elements of the vector space $\mathfrak{D}$. Looking closer at the commutators of the vectors defining the vector space $\mathfrak{D}$, we know that

$$
\left[\mathfrak{l}_{i}, \mathfrak{l}_{j}\right]=C_{m i j} \mathfrak{l}_{m}, i=1,2,3, m=1, \cdots, k
$$

where $C_{m i j}$ are the Lie algebra constants of the algebra $\mathfrak{g}$ and not all the coefficients $C_{m i j}, m=4, \cdots, k, i, j=1,2,3$, vanish. The linear combination of the first three vectors is in $\mathfrak{D}$. The remaining vectors $\mathfrak{l}_{i j}^{r}:=C_{m i j} \mathfrak{l}_{m}, m \geq 4$, although not in $\mathfrak{D}$, are elements of the algebra $\mathfrak{g}$ and can therefore be presented as a sum of an element from $\mathfrak{D}$ and an element from $T_{e}\left(G_{0}\right)$ (see footnote 4$)$. That is,

$$
\mathfrak{l}_{i j}^{r}=C_{m i j}^{r} \mathfrak{l}_{m}+\mathfrak{v}_{i j}
$$

where the vertical vector $\mathfrak{v}_{i j} \in T_{e}\left(G_{0}\right)$ and where the constants $C_{m i j}^{r}, i, j, m=$ $1,2,3$, are no longer the Lie algebra constants. In summary, for any pair of vectors $\mathfrak{l}_{i}, \mathfrak{l}_{j} \in \mathfrak{D} \subset \mathfrak{g}$, the corresponding commutator can be represented as

$$
\left[\mathfrak{l}_{i}, \mathfrak{l}_{j}\right]=C_{m i j} \mathfrak{l}_{m}+\left(C_{m i j}^{r} \mathfrak{l}_{m}+\mathfrak{v}_{i j}\right), \quad i, j, m=1,2,3 .
$$

Note, however, that as the first part of the decomposition is $G$ right invariant, the second part is not. Therefore, extending the vectors $\mathfrak{l}_{i}, i=1,2,3$, to the whole group $G$ by the right translation, we obtain that the corresponding vector fields $\tilde{\mathfrak{l}}_{i}$ have commutators which can be represented as

$$
\left[\tilde{\mathfrak{l}}_{i}(h), \tilde{\mathfrak{l}}_{j}(h)\right]=C_{m i j} \tilde{\mathfrak{l}}_{m}(h)+\left(C_{m i j}^{r}(h) \tilde{\mathfrak{l}}_{m}(h)+\mathfrak{v}_{i j}(h)\right), \quad i, j, m=1,2,3
$$

\footnotetext{
tThe distribution $\widetilde{\mathfrak{D}}: G \rightarrow T G$ defines a (right) invariant connection on the bundle $G \rightarrow G / G_{0} \cong$ $\mathbb{R}^{3}$, see [5]. The curvature of this connection is an invariant of the non-uniformly defective crystal states, the issue which we will look closer at in the forthcoming work.

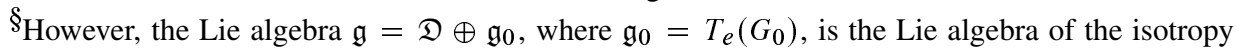
group $G_{0}$.
} 
where $h \in G$ and the coefficients $C_{m i j}$ are the Lie algebra constants. Indeed, although a bracket of two right invariant vector fields is right invariant and the right translations $d_{e} R_{h}$ are linear maps, the decomposition (4.5) of the tangent space $T G$ into the distribution $\tilde{\mathfrak{D}}$ and the tangent space to the cosets of the isotropy group $G_{0}$ is not. That is, when the right translations of the remainder vector $l_{i j}^{r}$ get decomposed, the decomposition is, in general, point $h$ dependent causing the coefficients $C_{m i j}^{r}(h)$ to be different at different points of the group $G$. Note that as the decomposition (4.5) is $G_{0}$ (isotropy group) right invariant, the coefficients $C_{m i j}^{r}(h)$ are constant along the fibers $T_{p}\left(p G_{0}\right), p \in G$. Note also that should the coefficients $C_{m i j}^{r}(h)$ be shown to be group element independent, the vector space $\mathfrak{D}$ would be a Lie subalgebra of the algebra $\mathfrak{g}$. (2.4).

The above analysis leads one to a straightforward generalization of the relation

Proposition 3. Consider a continuous lattice $l_{i}(x), i=1,2,3$, not necessarily uniformly defective, and let $S_{i j}(x)$ be its dislocation density tensor. Then

$$
\epsilon_{j k l} S_{i j}(x)=\hat{C}_{i k l}(x), \quad i, j, k=1,2,3, \quad x \in \mathbb{R}^{3}
$$

where

$$
\widehat{C}_{k i j}(x)=C_{k i j}+C_{k i j}^{r}(h)
$$

for $x=\Lambda(h, 0)$.

Indeed, the key point to consider is the fact that the Lie algebra homomorphism $d \lambda: \mathfrak{g} \rightarrow \mathcal{X}\left(\mathbb{R}^{3}\right)$ takes a Lie bracket into a Lie bracket of its image and that its kernel is the tangent space to cosets of the isotropy group $G_{0}$. Namely,

$$
d \lambda\left(\left[\mathfrak{l}_{i}, \mathfrak{l}_{j}\right]\right)(x)=d_{h} \Lambda_{0}\left(\left[\tilde{\mathfrak{l}}_{i}, \tilde{\mathfrak{l}}_{j}\right](x)=\left[l_{i}(x), l_{j}(x)\right]=\widehat{C}_{m i j}(x) l_{m}(x) .\right.
$$

The rest follows the argument of the uniformly defective case [3]. To see how this works in the case of a specific non-uniformly defective lattice, the reader may revisit Example 2 where the only nonzero coefficients $\widehat{C}_{k i j}(x)$ are $\widehat{C}_{113}(x)=\widehat{C}_{223}(x)=1$ and $\widehat{C}_{123}(x)=x_{2}$.

\section{REFERENCES}

[1] C. Davini, "A proposal for a continuum theory of defective crystals," Arch. Rational Mech. Anal., vol. 96, pp. 295-317, 1986.

[2] C. Davini and G. P. Parry, "A complete list of invariants for defective crystals," Proc. Roy. Soc. London A, vol. 432, pp. 341-365, 1991.

[3] M. Elżanowski and G. P. Parry, "Material symmetry in a theory of continuously defective crystals," J. Elasticity, vol. 74, pp. 215-237, 2004.

[4] V. V. Gorbatsevich, A. L. Onishchik, and E. B. Vinberg, Lie groups and Lie algebras I, ser. Encyclopedia of Mathematical Sciences. Berlin Heilderberg: Springer-Verlag, 1993, vol. 20. 
[5] S. Kobayashi and K. Nomizu, Foundations of Differential Geometry. New York: John Wiley \& Sons, Inc., 1996.

[6] R. S. Palais, "A global formulation of the lie theory of transformation groups," Mem. Amer. Math. Soc., vol. 22, 1957.

[7] G. P. Parry, "Group properties of defective crystal structures," Mathematics and Mechanics of Solids, vol. 8, pp. 515-538, 2003.

[8] G. P. Parry and R. Sigrist, "Reconciliation of local and global symmetries for a class of crystals with defects," J. Elasticity, vol. 107, pp. 81-104, 2012.

Authors' addresses

Marek Z. Elżanowski

Fariborz Maseeh Department of Mathematics and Statistics, Portland State University, Portland, OR 97207, USA

E-mail address: elzanowskim@pdx.edu

\section{Serge Preston}

Fariborz Maseeh Department of Mathematics and Statistics, Portland State University, Portland, OR 97207, USA

E-mail address: sergeepdx.edu 